\title{
The influence of heart coherence on synchronization between human heart rate variability and geomagnetic activity
}

\author{
Rollin McCraty ${ }^{1}$, Mike Atkinson ${ }^{2}$, Inga Timofejeva ${ }^{3}$, Roza Joffe ${ }^{4}$, Alfonsas Vainoras ${ }^{5}$, \\ Mantas Landauskas $^{6}$, A. Abdullah Alabdulgader ${ }^{7}$, Minvydas Ragulskis ${ }^{8}$ \\ ${ }^{1,2}$ HeartMath Institute, Boulder Creek, CA 95006, USA \\ ${ }^{3,6,8}$ Department of Mathematical Modelling, Kaunas University of Technology, Kaunas, 51368, Lithuania \\ ${ }^{4}$ Health Research Institute, Lithuanian University of Health Sciences, Kaunas, 50009, Lithuania \\ ${ }^{5}$ Cardiology Institute, Lithuanian University of Health Sciences, Kaunas, 44307, Lithuania \\ ${ }^{7}$ Director of Research and Scientific Bio-Computing, Prince Sultan Cardiac Center, \\ Alhasa, Hofuf, 31982, Saudi Arabia \\ ${ }^{3}$ Corresponding author \\ E-mail: ${ }^{1}$ rollin@heartmath.org, ${ }^{2}$ mike@heartmath.org, ${ }^{3}$ inga.timofejeva@ktu.edu, ${ }^{4}$ joffero@gmail.com, \\ 5alfavain@gmail.com, ${ }^{6}$ mantas.landauskas@ktu.lt, ${ }^{7}$ kidsecho@yahoo.com, ${ }^{8}$ minvydas.ragulskis@ktu.lt \\ Received 12 September 2018; received in revised form 4 December 2018; accepted 27 December 2018 \\ DOI https://doi.org/10.21595/chs.2018.20480 \\ Check for updates \\ Copyright (C) 2018 Rollin McCraty, et al. This is an open access article distributed under the Creative Commons Attribution License, \\ which permits unrestricted use, distribution, and reproduction in any medium, provided the original work is properly cited.
}

\begin{abstract}
Results of a study on the effect of the Heart Lock-In meditation technique on the synchronization between heart rate variability and local magnetic field activity is presented in this paper. A technique based on the near-optimal chaotic attractor embedding was applied in order to evaluate the geometrical synchronization between analyzed time series. The results demonstrate that Heart Lock-In technique had a strong influence on the relationship between cardiac and geomagnetic activity.
\end{abstract}

Keywords: Earth's magnetic field, geomagnetic field, heart rate variability, HRV, ANS, nonlinear dynamical systems.

\section{Introduction}

All biological systems, which are embedded within the Sun and Earth's magnetospheres, are exposed to invisible fluctuating magnetic fields that span a wide range of frequencies [1]. It is well known that geomagnetic field line resonances and the Schumann resonances, which exist in the cavity between Earth and the ionosphere, generate a range of resonant frequencies that directly overlay those of the human brain, autonomic nervous system (ANS), and cardiovascular system. Of all the physiological systems studied, the rhythms produced by the brain and heart are most affected by changes in geomagnetic conditions [2-8]. It has been demonstrated that numerous physiological rhythms are affected by and can synchronize with solar and geomagnetic activity [9-12]. However, several recent studies that utilized continuous monitoring of heart rate variability (HRV) over longer time periods have shown that ANS activity can synchronize to a surprising degree with changes in the amplitude of resonant frequencies produced by geomagnetic field-line resonances, Alfvén waves and the Schumann resonances [13, 14].

HRV is the measurement of the time intervals between adjacent pairs of heartbeats, which reflects autonomic nervous system dynamics [15]. The amount of naturally occurring HRV one has reflects the functional status of codependent regulatory systems that operate over different time scales to adapt to environmental and psychological challenges [16]. Lower levels of variation in age-adjusted HRV indicate pathology, chronic stress, or insufficient functioning in regulatory systems in the neuro axis and is associated with of all-cause mortality [17-19]. Higher levels of $\mathrm{HRV}$ are associated with resiliency, capacity to self-regulate and to adapt to changing demands $[16,19]$.

Several studies have found that an intensification in field-line resonances can lead to alterations 
in cardiovascular function and it has been suggested that this may be due to the fact that many of the field line resonances have frequencies that directly overlap with those of the ANS and cardiovascular system $[9,12,13,20]$.

A previously conducted study provided evidence suggesting that participant's psychological state and quality of interactions between group members was related the degree of HRV synchronization with the magnetic field data and other participants in the group [14]. It has also been proposed that when one is in a state called physiological or HRV coherence that one is more likely to be coupled to and therefore synchronized to the Earth's magnetic fields [21]. In order to test this hypothesis, we also examined the potential effects of a heart focused mediation technique called the Heart Lock-In, which increases the coherence in one's heart rhythms, and has been shown to increase the synchronization of heart rhythms between participants in a group setting [22].

In order to improve the assessment of physiological synchronization and identify individuals' response patterns, we utilized a newly developed and validated analysis approach using near-optimal chaotic attractor embedding techniques. This allowed us to identify specific patterns of synchronization between heart rate variability and local magnetic field data, and assess potential effects of participants being in a state of HRV coherence on synchronization in a group of people located in Lithuania.

\section{Methods and procedures}

\subsection{Participants}

The experiment location had a local site coordinator who was responsible for participant recruitment, logistics coordination and participant training in the study procedures and use of the HRV recorders. Study coordinator in Lithuania recruited a total of 20 participants. The mean age (sd) of the 20 participants in the analysis was 23.3 (0.6) years consisting of 16 females and 4 males.

During the 14 day period between 26 February and 12 March 2015, 20 participants located in Lithuania participated in the study. All of the participants were healthy and either worked or attended classes during daytime hours. The Lithuanian group consisted of 20 medical students attending the Lithuanian University of Health Sciences.

\subsection{Ethics statement}

The research met all applicable standards for the ethics of experimentation in accordance with the Declaration of Helsinki. The permit to perform biomedical investigation was granted by the Kaunas Regional Ethics Committee for Biomedical Investigations, No. BE-2-51, 23.12.2015 (copies of documents are enclosed as supplemental data). Participants provided written informed consent prior to the experiment.

\subsection{HRV data collection}

HRV is a noninvasive measure that reflects ANS activity and dynamics. All participants underwent daily 24-hour ambulatory HRV recordings during a two-week period between 26 February and 12 March 2015 (Bodyguard2, Firstbeat Technologies Ltd., Jyväskylä, Finland). Prior to the start of the study, each participant received instructions on attaching, starting, and stopping the recorders as well as instructions to participants for logging day to day activity, sleep, waking, etc. from the local site coordinator.

The group coordinator was instructed in how to retrieve data from the HRV recorders and upload it to the data collection FTP site. Participants were instructed to stop the recorder each morning after waking up to start the day, and allowed up to 50 minutes to shower or bathe before reattaching the recorder and starting the new day's recording. Ambu Blue Sensor VL microporous 
breathable disposable electrodes were used for all of the recordings. The electrodes were placed in a modified V5 position. To minimize skin irritation over the two weeks, participants were encouraged to locate the electrodes around three different positions near the V5 electrode sites. The HRV recorder calculates the RR Interval ( $R$ is a point corresponding to the peak of the $Q R S$ complex of the ECG wave; and RR is the interval between successive Rs) from the electrocardiogram sampled at $1000 \mathrm{~Hz}$. The RR interval data were stored locally in the device memory, and downloaded to a computer workstation at the completion of the study.

All of the HRV recordings were downloaded from the FTP site to a computer workstation and analyzed using DADiSP 6.7. Inter-Beat-Intervals greater or less than $30 \%$ of the mean of the previous four intervals were considered artifacts, and were removed from the analysis record. Following an automated editing procedure, all of the recordings were manually reviewed by an experienced technician, and, if needed, corrected. Daily recordings were processed in consecutive 5-min segments in accordance with the standards established by the HRV Task Force. Any 5-min segment with $>10 \%$ of the IBIs either missing or removed in editing were excluded from the analysis. The local time stamps in the HRV recordings were converted to Coordinated Universal Time (UTC) to enable synchronization between locations and magnetic field data sets.

\subsection{Magnetic field data}

The local magnetic field intensity was measured using magnetometer located in Lithuania which is a part of the Global Coherence Monitoring Network [21]. Two magnetic field detectors (Zonge Engineering ANT-4) at the site are positioned in the north-south and east-west axes to detect local time-varying magnetic field strengths (sensitivity $1 \mathrm{pT}$ ) over a wide frequency range $(0.01-300 \mathrm{~Hz})$ while maintaining a flat frequency response. The data acquisition infrastructure captures, then stamps, the global positioning system time, and transmits the data to the common server. Each magnetometer in the network is continuously sampled at a rate of $130 \mathrm{~Hz}$.

\subsection{Heart Lock-In procedure}

On March 5th, the group participated in the Heart Lock-In technique for a 15-minute period. The Heart Lock-In technique focuses on building the capacity to sustain heartfelt positive emotions. The technique is generally practiced for five to fifteen minutes at a time, although longer sessions may be used as well. The steps of Heart Lock-In are:

Step 1: Focus your attention in the area of the heart. Imagine your breath is flowing in and out of your heart or chest area, breathing a little slower and deeper than usual.

Step 2: Activate and sustain a regenerative feeling such as appreciation, care or compassion.

Step 3: Radiate that renewing feeling to yourself and others [23].

Use of this technique is typically accompanied by feelings of peacefulness, harmony, and a sense of inner warmth, and is often an effective means to diffuse accumulated stress and negative feelings [24].

\subsection{Computation of the power of the local magnetic field}

The spectral power of the local magnetic field was computed using the magnetic field intensity values and applying the algorithm denoted in [14] as Algorithm A. The main steps of the algorithm included the computation of the spectrogram of the magnetic field intensity data signal (for one-second time intervals), clipping the spectrogram to a certain level in order to minimize the noise (manmade noise, lightning) in the magnetic field data and summing the values over the frequency range $[0,1] \mathrm{Hz}$. Empirically obtained optimal noise clipping level was $1.25(\mathrm{pT})^{2} / \mathrm{Hz}$. 


\subsection{Determination of geometrical synchronization between participants' HRV and local magnetic field power time series}

For the determination of synchronization between participants' HRV and the signal of the power of local magnetic field, computed for 1s time intervals (see Section 2.6), we employed techniques developed, validated and described in detail in [14]. This approach is based on the fact, that it is possible to map a data signal into an integer which describes the geometrical dynamics of the data signal. The integer corresponds to the optimal time lag value that maximizes the area of the attractor constructed from the data series.

We slightly modified the algorithm described in [14] as Algorithm $C$ to be relevant to this study to the following procedure:

Let $R R=\left(R R_{1}, \ldots, R R_{n}\right)$ and $M=\left(M_{1}, \ldots, M_{n}\right)$ be synchronously sampled participant's HRV and local magnetic field (MF) power signals of size $n$.

1) Divide series $R R$ and $M$ into $T$ 5-minute-long observation windows (according to HRV analysis standards [25]) and compute optimal time lag values for each observation window for $\mathrm{HRV}$ time series $\left(\tau_{*}^{(R R)}=\left(\tau_{* 1}^{(R R)}, \ldots, \tau_{* T}^{(R R)}\right)\right)$ and magnetic field power data signal $\left(\tau_{*}^{(M)}=\left(\tau_{* 1}^{(M)}, \ldots, \tau_{* T}^{(M)}\right)\right)$. This step reduces the analyzed HRV and MF time series to integer vectors, characterizing geometrical features of those series.

2) Smooth obtained optimal time lag vectors in order to identify averaged changes in time lags for $\operatorname{HRV}\left(\bar{\tau}_{*}^{(R R)}\right)$ and magnetic field data $\left(\bar{\tau}_{*}^{(M)}\right)$.

3) Calculate the Pearson correlation coefficient between the obtained mean time lag vectors $C^{(R R, M)}=\rho\left(\bar{\tau}_{*}^{(R R)}, \bar{\tau}_{*}^{(M)}\right)$.

Consider a set of HRV time series $R R^{(k)}=\left(R R_{1}^{(k)}, \ldots, R R_{n}^{(k)}\right), k=\overline{1, K}$, corresponding to $K$ participants. In order to calculate the mean synchronization between the HRV of all $K$ participants and magnetic field, we compute HRV/Magnetic field correlation coefficients $C^{\left(R R^{(k)}, M\right)}$ for each participant $k=\overline{1, K}$ and calculate the mean correlation coefficient $C^{\left(R R^{(1 . K)}, M\right)}=\frac{1}{K} \sum_{k=1}^{K} C^{\left(R R^{(k)}, M\right)}$.

\subsection{Identification of the effect of being in a coherent state using the Heart Lock-In technique on synchronization with the magnetic field}

Procedures for the estimation of synchronization between participants' HRV and local magnetic field power time series, described in Section 2.7, were applied to the data described above.

Since the Heart Lock-In technique took place once for a 15-minute period (on March 5th, 2015), we calculated participants' HRV/MF synchronization for each day of the study separately in order to see if the synchronization between participants' HRV and magnetic field activity on the day of the Heart Lock-In was different compared to days before and after the using the technique.

\section{Results}

The results of the analysis of the synchronization between participants' $\mathrm{HRV}$ and magnetic field activity are presented in Fig. 1. Each figure consists of plots displaying the synchronization between each participant's HRV and magnetic field activity for six days $(2015 / 03 / 01$ - 2015/03/06). The Fig. 1(e) part of each figure corresponds to the day of the application of the Heart Lock-In technique.

Fig. 2 depicts the mean synchronization between groups' HRV and magnetic field power for each day of the experiment (red bar corresponds to the day the participants did the Heart Lock-In). 


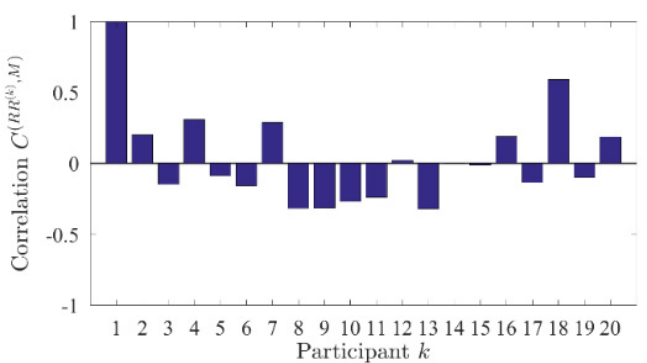

a) Day 2015.03.01

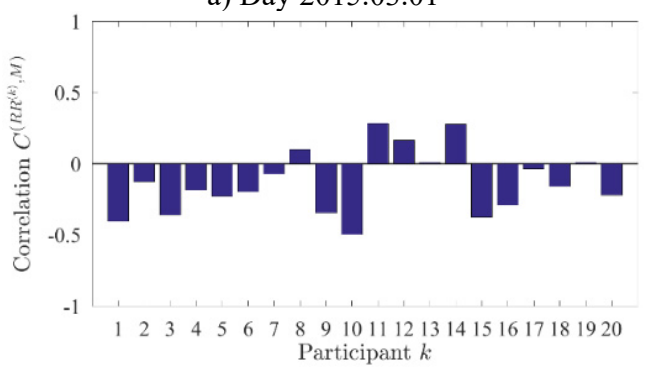

c) Day 2015.03 .03

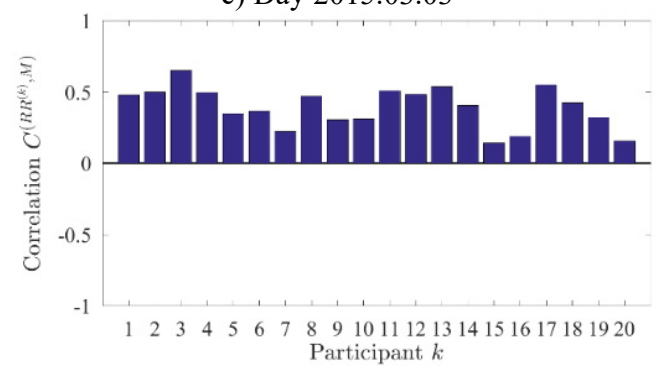

e) Day of heart lock-in

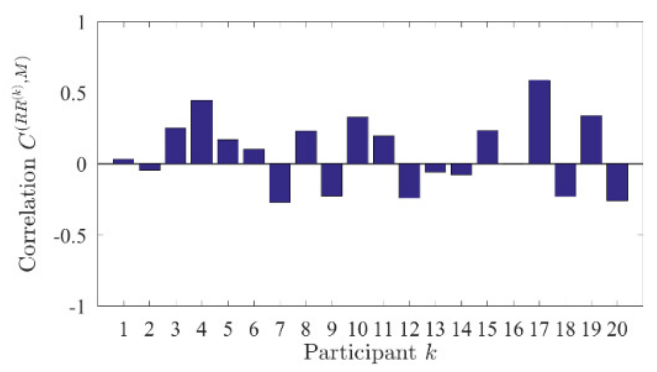

b) Day 2015.03 .02

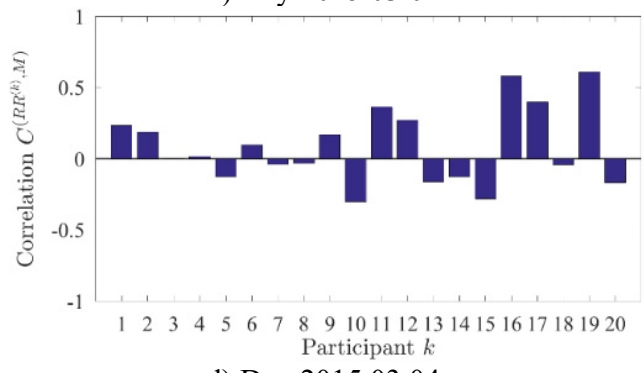

d) Day 2015.03.04

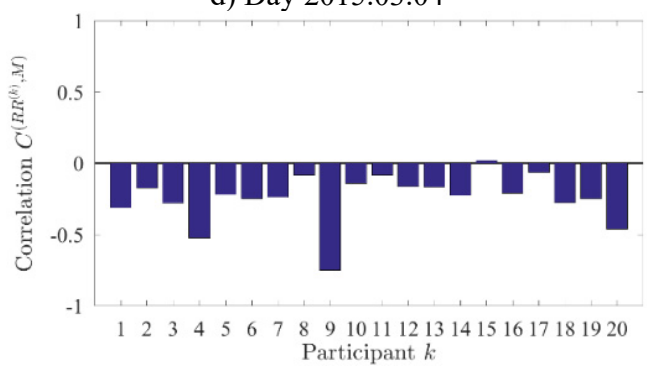

e) Day 2015.03.06

Fig. 1. The results of the analysis of the HRV/MF synchronization.

Each part of the figure displays participants' HRV/MF synchronization for the indicated day. Part (e) corresponds to the day of the Heart Lock-In

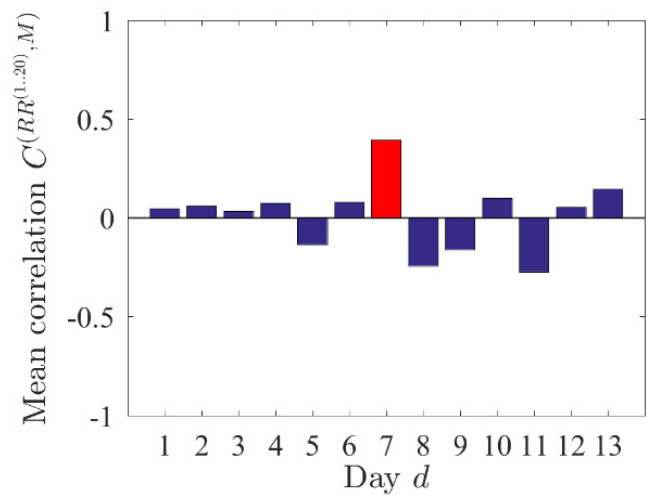

Fig. 2. Groups' mean HRV/MF synchronization for each day of the experiment

It can be seen that the synchronization (positive correlation) between participants' HRV and magnetic field activity (Fig. 2) on the day of the Heart Lock-In was the highest. Furthermore, all participants were highly positively correlated/synchronized with the local magnetic field during the day of the application of the Heart Lock-In technique (Fig. 1(f)) which contrasted with the synchronization results for days before and after the training. 
THE INFLUENCE OF HEART COHERENCE ON SYNCHRONIZATION BETWEEN HUMAN HEART RATE VARIABILITY AND GEOMAGNETIC ACTIVITY. Rollin McCraty, Mike AtKinson, Inga TimofEJEVA, RoZA JofFe, AlFonsAs VAinORAs, MANTAS LANDAUSKAS, ET AL.

\section{Conclusions}

A technique based on the near-optimal chaotic attractor embedding was applied in order to evaluate the geometrical synchronization between analyzed time series. The results of the conducted study indicate that the applied Heart Lock-In meditation technique has a positive impact on the synchronization between the human heart rate variability and the Earth's magnetic field. Thus, such medical techniques are shown to be valuable not only psychologically (in sustaining positive emotions), but also physiologically since high synchronization between heart rate variability and geomagnetic activity has been associated with better health conditions. Further study of these effects using different computational and experimental techniques are a definite objective of future research.

\section{References}

[1] Cornelissen G., et al. Chronomes, time structures, for chrono bioengineering for "a full life". Biomedical Instrumentation and Technology, Vol. 33, 1999, p. 152-187.

[2] Otsuka K., Cornelissen G., Norboo T., Takasugi E., Halberg F. Chronomics and "glocal" (Combined Global and Local) assessment of human life. Progress of Theoretical Physics Supplement, Vol. 173, 2008, p. 134-152.

[3] Dimitrova S., Stoilova I., Cholakov I. Influence of Local Geomagnetic Storms on Arterial Blood Pressure. Bioelectromagnetics, Vol. 25, 2004, p. 408-414.

[4] Hamer J. R. Biological Entrainment of the Human Brain by Low Frequency Radiation. Northrop Space Labs, 1965, p. 65-199.

[5] Oraevskii V. N., Breus T. K., Baevskii R. M., Rapoport S. I., Petrov V. M., Barsukova Zh. V., Gurfinkel Iu. I., Rogoza A. T. Effect of geomagnetic activity on the functional status of the body. Biofizika, Vol. 43, 1998, p. 819-826.

[6] Pobachenko S. V., Kolesnik A. G., Borodin A. S., Kalyuzhin V. V. The contingency of parameters of human encephalograms and Schumann resonance electromagnetic fields revealed in monitoring studies. Complex Systems Biophysics, Vol. 51, 2006, p. 480-483.

[7] Cornélissen G., et al. Non-photic solar associations of heart rate variability and myocardial infarction. Journal of Atmospheric and Solar-Terrestrial Physics, Vol. 64, 2002, p. 707-720.

[8] Cornelissen G., McCraty R., Atkinson M., Halberg F. General differences in circadian and extra-circadian aspects of heart rate variability (HRV). 1st International Workshop of The TsimTsoum Institute, Poland, 2010.

[9] Doronin V. N., Parfentev V. A., Tleulin Zh S., Namvar R. A., Somsikov V. M., Drobzhev V. I., Chemeris A. V. Effect of variations of the geomagnetic field and solar activity on human physiological indicators. Biofizika, Vol. 43, 1998, p. 647-653.

[10] Halberg F., Cornelissen G., Mccraty R., Al-Abdulgader A. Time structures (chronomes) of the blood circulation, populations' health, human affairs and space weather. World Heart Journal, Vol. 3, 2011.

[11] Khorseva N. Using psychophysiological indices to estimate the effect of cosmophysical factors (Review). Izvestiya, Atmospheric and Oceanic Physics, Vol. 49, 2013, p. 839-852.

[12] Khabarova O., Dimitrova S. On the nature of people's reaction to space weather and meteorological weather changes. Sun and Geosphere, Vol. 4, 2009, p. 60-71.

[13] McCraty R., et al. Synchronization of human autonomic nervous system rhythms with geomagnetic activity in human subjects. Journal of Environmental Research and Public Health, Vol. 14, 2017, p. 770.

[14] Timofejeva I., et al. Identification of a group's physiological synchronization with earth's magnetic field. International Journal of Environmental Research and Public Health, Vol. 14, 2017, p. 2-22.

[15] Shaffer F., McCraty R. Zerr C. A healthy heart is not a metronome: An integrative review of the heart's anatomy and heart rate variability. Frontiers in Psychology, Vol. 5, 2014, p. 1040.

[16] McCraty R., Shaffer F. Heart rate variability: new perspectives on physiological mechanisms, assessment of self-regulatory capacity, and health risk. Global Advances in Health and Medicine: Improving Healthcare Outcomes Worldwide, Vol. 4, 2015, p. 46-61.

[17] Tsuji H., et al. Reduced heart rate variability and mortality risk in an elderly cohort. The Framingham Heart Study. Circulation, Vol. 90, 1994, p. 878-883. 
[18] Singer D. H., et al. Low heart rate variability and sudden cardiac death. Journal of Electrocardiology, Vol. 21, 1988, p. 46-55.

[19] Thayer J. F., Hansen A. L., Saus-Rose E., Johnsen B. H. Heart rate variability, prefrontal neural function, and cognitive performance: the neurovisceral integration perspective on self-regulation, adaptation, and health. Annals of Behavioral Medicine, Vol. 37, 2009, p. 141-153.

[20] Zenchenko T., Medvedeva A., Khorseva N., Breus T. Synchronization of human heart-rate indicators and geomagnetic field variations in the frequency range of 0.5-3.0 $\mathrm{mHz}$. Izvestiya, Atmospheric and Oceanic Physics, Vol. 50, 2014, p. 736-744.

[21] McCraty R., Deyhle A. Bioelectromagnetic and Subtle Energy Medicine. Second Edition, CRC Press, 2015.

[22] McCraty R. New frontiers in heart rate variability and social coherence research: techniques, technologies, and implications for improving group dynamics and outcomes. Frontiers in Public Health, Vol. 5, 2017, p. 267-2.

[23] McCraty R., Moor S., Goelitz J., Lance S. Transforming Stress for Teens: The HeartMath Solution for Staying Cool Under Pressure. New Harbinger, 2016.

[24] McCraty R., Tomasino D. Stress in Health and Disease. Wiley-VCH, 2006.

[25] Novak V., Saul J. P., Eckberg D. L. Task force report on heart rate variability. Circulation, Vol. 96, 1997, p. 1056-1057. 